\title{
EFFECTIVENESS OF BALANCE AND TAI CHI EXERCISE IN EARLY TYPE II DIABETIC AMONG FEMALE POPULATION
}

\author{
RAJALAXMI $\mathrm{V}^{1 *}$, VIJAYA PRIYA $\mathrm{V}^{1}$, RAMACHANDRAN $S^{1}$, LATHA $S^{2}$ \\ ${ }^{1}$ Faculty of Physiotherapy, Dr. M.G.R. Educational and Research Institute University, Chennai - 600 077, Tamil Nadu, India. ${ }^{2}$ Department \\ of Physiotherapy, Sri Venkateswara College of Physiotherapy, Puducherry, India.
}

Email: rajalaxmigopalakrishnan@gmail.com

Received: 22 December 2017, Revised and Accepted: 02 February 2018

ABSTRACT

Objective: India is considered to be a diabetic capital as many Indians are diabetic. The common treatment for diabetes is medication. The purpose of this study is to bring out the other remedial measures for diabetic treatment and to create awareness among the people about the alternative measures. This study is about the effect of balancing exercise and tai chi exercises for early diabetic. It is, therefore, possible that an individual can experience a noticeable decreased in blood glucose following a balancing exercise and modified tai chi program. This study is to find out the effect of balancing exercise and tai chi program and to compare the effectiveness.

Methods: Once the study is approved by the institutional review board, 50 samples from 65 volunteers with Type 2 diabetes between the age group of 25-35 years were selected from the ACS Medical College and Hospitals based on the inclusion criteria. After getting the consent, the samples were equally divided into two groups; Group A: Balancing exercise and Group B: Tai chi exercise. Both groups received intervention for 8 weeks 6 days/ week 2 sessions/day. Pre- and post-test was done using the outcome measures of blood glucose levels (glucometer), body mass index, and waist circumference (inch tape).

Result: On comparing the mean values of waist circumference and blood glucose level between Group A and B, both the groups showed significant reduction in the post-test values. However, Group B tai chi for diabetes shows the mean (34.4) which is effective than the Group A (38.5) for waist circumference (in inches) and also Group B (156.70) is effective than the Group A (184.10) for blood glucose level (mg/dL) at $\mathrm{p} \leq 0.05$.

Conclusion: The study concluded that the balancing exercise and tai chi exercise are effective in the reduction of blood glucose level and waist circumference. However, tai chi is more effective than the balancing exercise.

Keywords: Balance exercise, Tai chi, Blood glucose level.

(C) 2018 The Authors. Published by Innovare Academic Sciences Pvt Ltd. This is an open access article under the CC BY license (http://creativecommons. org/licenses/by/4. 0/) DOI: http://dx.doi.org/10.22159/ajpcr.2018.v11i5.24346

\section{INTRODUCTION}

India is the diabetes capital at home to $69.1 \%$ of diabetic patients. Indians were the second highest in the incidence rate after China [1]. It is possible to stop or delay the onset of Type 2 diabetes (T2D) by reducing lifestyle risk factors through moderate weight loss and increased physical activity. Several studies have shown the lifestyle changes that include exercise can significantly delay and possibly prevent diabetes [2]. If good glucose control is not achieved overtime, prolonged hyperglycemia can lead to destructive and several outcomes such as retinopathy, nephropathy, neuropathy, cardiovascular disease, stroke, pressure ulcers neuropathic wounds loss of peripheral protective sensation gangrene, limb amputation, and death.

Balancing a body requires coordination of input from multiple sensory systems including the vestibular, somatosensory, skeletal system (muscles, joints, and their sensors), and visual systems. It has a possible impact of reducing the glucose level in the blood. Tai chi is just an internal Chinese martial art practiced for both its defense training and its health benefits. Tai chi has been introduced to health professionals as a moderate intensity aerobic exercise. The training of tai chi involves to primary features: Taolu - slow sequence of movements and tuishou for training movement principles of the form with a partner, the modified tai chi program for people with poorly controlled T2D reduces the glycemic level and enhance physical and social functions [3].

Hence, the balancing exercise and tai chi exercise believed to have an impact in reducing the blood glucose level. The purpose of this study is to determine the extent of effectiveness of balancing exercise and tai chi program on diabetes and to compare their effects.

\section{METHODS}

Once the study is approved by an institutional review board, 50 participants were selected among 65 volunteers from ACS Medical College and Hospital, Chennai, with the age group of 25-35 years (mean age-31.8). After pre-test measurements of glycemic level and waist circumference, participants were randomized into two groups (A and B). The samples were included if they were aged between 25 and 35 years had glycosylated hemoglobin (HbA1C) levels for the past 2 years. They were also obliged to be sedentary, defined as reporting never having participated in the structured exercise program or recreational physical activity. Subjects were excluded if they had uncontrolled DM above 3 years, congestive cardiac failure, unstable angina, acute myocardial infarction, proliferative diabetic retinopathy, uncontrolled hypertension, and renal insufficiency. The subjects were fully explained about the benefits of participating in the study and asked to fill the consent form in acceptance with participation of the study which is duly signed by the participants and the researcher.

Group A performed balancing exercise program involving single limb stance, heel to toe, dynamic walking, knee marching and body circle repeated for 10 sets $/ 2$ sessions for about $40 \mathrm{~min}$ in the 2 weeks, and progressed to the repetition of 20 sets/ 2 sessions in the next 2 weeks. The exercise was progressed from a stable platform to unstable 
platform with a form-based support and wobble board along with upper extremity exercises combined with perturbations for the next 4 weeks. Group B received tai chi for diabetes (TCD) program developed by Lam (based on a standardized form of 21 movements from the combined Yang and sun style of tai chi). It consists of $5 \mathrm{~min}$ of warm-up exercises, $40 \mathrm{~min}$ of tai chi movements, and finally $5 \mathrm{~min}$ of cool down exercise. Group B received exercise program twice a day for 8 weeks. Various motivation strategies were applied to reduce the dropout rate, such as drawing up the contract to encourage the patients to perform exercise regularly at home, providing tai chi music tape and thrice a week follow-up calls to remind the participants of the exercise. The outcomes measured were the blood glucose level using the portable glucometer and the waist circumference using the inch tape.

\section{Data analysis}

All participants with follow-up data were analyzed using SPSS software according to their group allocation. The difference in change from pre- to the post-intervention between the balancing exercise group and tai chi program group for each outcome was evaluated using an independent t-test.

\section{RESULT}

After discussing the collected data, the comparison is done between the Groups A and B in waist circumference and blood glucose level. The pre-test mean value for waist circumference in Group A is 39.5. After giving them a balancing exercise for 8 weeks, the waist circumference reduced to 1-2 inches only in some individuals, and thereby the post-test mean value is 38.5. Group B also underwent for waist circumference measurement before and after the tai chi exercise. The pre-test mean value is 38.1 and they performed tai chi for 8 weeks and showed improvement in the reduction of waist circumference for about 1-2 inches in almost all, where the post-test mean value is 34.4 .

Table 1 summarizes the mean and standard deviation (SD) of pre- and post-test along with the values of t-test and significance of waist circumference.

Table 2 summarizes the mean and SD of pre- and post-test along with the values of t-test and significance of blood glucose level.

The pre- and post-test data are analyzed for the blood glucose level between Groups A and B. The pre-test and post-test mean value of blood glucose level in Group A is 190.70 and 184.10, respectively. This shows that there is a considerable reduction in blood glucose after performing a balancing exercise. The pre-test and post-test value of blood glucose in Group B is 179.30 and 156.70, respectively, showing that tai chi has a good effect in reducing the blood glucose level.

Table 1: Comparison of waist circumference between Groups A and $B$ in pre- and post-test

\begin{tabular}{lllll}
\hline \multirow{2}{*}{$\begin{array}{l}\text { Waist } \\
\text { circumference }\end{array}$} & ${ }^{*}$ Group A & ${ }^{*}$ Group B & \multirow{2}{*}{ t-test } & Significance \\
\cline { 2 - 3 } & Mean \pm SD & Mean \pm SD & & \\
\hline Pre-test & $39.5 \pm 2.41$ & $38.7 \pm 2.05$ & 0.79 & 0.436 \\
Post-test & $38.5 \pm 3.06$ & $34.4 \pm 1.95$ & 3.56 & 0.000 \\
\hline
\end{tabular}

SD: Standard deviation

Table 2: Comparison of blood glucose level between Groups A and $B$ in pre- and post-test

\begin{tabular}{lllll}
\hline $\begin{array}{l}\text { Blood } \\
\text { glucose }\end{array}$ & ${ }^{*}$ Group A & $*$ Group B & \multirow{2}{*}{ t-test } & Significance \\
\cline { 2 - 3 } & Mean \pm SD & Mean \pm SD & & \\
\hline Pre-test & $190.70 \pm 21.09$ & $179.30 \pm 26.63$ & 1.06 & 0.303 \\
Post-test & $184.10 \pm 21.68$ & $156.70 \pm 25.21$ & 2.06 & 0.000 \\
\hline
\end{tabular}

SD: Standard deviation
On comparing the mean values of waist circumference and blood glucose level between Group A and B, analysis shows significant reduction in the post-test values of both the groups. However, Group B TCD shows (34.4) mean which is effective than the Group A (38.5) for waist circumference and also Group B (156.70) is effective than the Group A (184.10) on blood glucose level at $\mathrm{p} \leq 0.05$.

\section{DISCUSSION}

The present study was conducted to compare and find out the effect of balancing exercise and tai chi program for diabetes among the diabetic individual with the mean age of 32 years. It was noticed that balancing exercise and tai chi program showed improvement for DM Type 2. In previous studies, diabetic patient who practiced tai chi exercise for 6 months reported a higher reduction in the HbA1C level and good control of blood glucose $[3,4]$. The pre-test mean value for waist circumference in Group A is 39.5. After giving them a balancing exercise for 8 weeks, the waist circumference reduced to 1-2 inches only in some individuals, and thereby the post-test mean value is 38.5 . Group B also underwent for waist circumference measurement before and after the tai chi exercise. The pre-test mean value is 38.1 and they performed tai chi for 8 weeks and showed improvement in the reduction of waist circumference for about 1-2 inches in almost all, where the post-test mean value is 34.4 .

The pathophysiology of DM is multifactorial. T2DM is a non-insulin dependent. DM where it is because of impaired insulin secretion and insulin resistance. Exercise promotes the increased uptake of glucose and increased insulin sensitivity, thereby lowers the blood glucose level [5]. Balancing exercise requires coordination of visual, vestibular, and skeletal muscle work. More energy and good proprioception are required to maintain good balance. Hence, balancing exercise enhances glucose uptake by the cells to a considerable amount. The low-to-moderate intensity of tai chi exercise is expected to improve the glucose metabolism, resulting in lower levels of $\mathrm{HbA1C}$ and insulin resistance [6]. A number of studies conducted so far on positive effects of exercise and physical activity on insulin sensitivity and glycemic control [7,8]. Aerobic exercise and resistance training has been proven in controlling the blood glucose level [9].

Tai chi is a Chinese martial art which is said to have a beneficial role in controlling the blood glucose level by Lam et al. in 2008 . Balance exercise is an exercise which improves balance that requires coordination of many muscle groups. This study correlates with the study done to find the tai chi program effect on glycemic control by Lam et al. and Song et al. Even tai chi is said to be a type of meditation that improves depression which is common in Type 1 and T2D [10]. Further, findings can be done to find out the effect of tai chi in depression of diabetic individuals.

From our findings, the pre-test and post-test mean values of waist circumference and blood glucose level in Groups A and B show that both groups have a statistically considerable significant difference at $\mathrm{p} \leq 0.05$. Secondarily, the Group B (tai chi) is effective when compared to Group A (balancing exercise). Further studies can be done with other alternative exercises and therapies, the duration of the exercises can also be increased to find out the long-term effects of exercise, the outcome can also be measured immediately after the treatment session daily to find out the immediate effects on blood glucose.

This study states that T2D is a lifelong ailment, thus prolonged exposure to drugs further deteriorates the quality of life of the patient. Further, the cost of the prolonged treatment only adds to the woes of the patient. There is an urgent need to come up with complementary treatments that can be used as therapy along with the drugs to reduce the dependence on the drugs and better manage the disease. Daily exercise, inclusion of nutritious diet and vitamin, and nutraceutical supplements in everyday life can significantly 
improve the well-being of diabetic patients [11]. The study concludes the effect of physical exercise on the dynamics of glucose and insulin to confirm the role of physical exercise as a prevention for subjects at risk, to stress the benefit that can be gained by T2DM from improving SI index. The goal for all subjects with diabetes should be to learned their individual glycemic responses to exercise and to control glucose fluctuations by modifying insulin dosage diet appropriately [12].

\section{CONCLUSION}

The result of this study reveals that there is a significant difference in the post-test values of both the groups. The study concluded that the balancing exercise and tai chi exercise are effective in the reduction of blood glucose level and waist circumference. However, tai chi is more effective than the balancing exercise.

\section{ACKNOWLEDGMENT}

The authors would like to thank the authorities of Dr. MGR Educational and Research Institute, University and the Principal Faculty of Physiotherapy for providing our facilities required to conduct the study.

\section{ETHICAL CONSIDERATIONS}

The manuscript is approved by the institutional review board of faculty of physiotherapy. All the procedures were performed in accordance with the ethical standards of the responsible ethics committee of both (institutional and national) on human experimentation and the Helsinki Declaration of 1964 (as revised in 2008).

\section{FUNDING}

This is a self-funded study.

\section{CONFLICT OF INTEREST}

All contributing authors declare that they have no conflicts of interest. This study was approved by the Institutional Review Board of
Physiotherapy, Dr. MGR Educational and Research Institute University, Chennai.

\section{REFERENCES}

1. Tripathy JP, Thakur JS, Jeet G, Chawla S, Jain S, Pal A, et al. Prevalence and risk factors of diabetes in a large community-based study in north India: Results from a STEPS survey in Punjab, India. Diabetol Metab Syndr 2017;9:8

2. Tudor-Locke CE, Bell RC, Meyers AM. Revisiting the role of physical activity and exercise in the treatment of type 2 diabetes. Can J Appl Physiol 2000;25:466-92.

3. Lam P, Dennis SM, Diamond TH, Zwar N. Improving glycaemic and BP control in Type 2 diabetes. The effectiveness of tai chi. Aust Fam Physician 2008;37:884-7.

4. Song R, Ahn S, Roberts BL, Lee EO, Ahn YH. Adhering to a t'ai chi program to improve glucose control and quality of life for individuals with Type 2 diabetes. J Altern Complement Med 2009;15:627-32.

5. Wei M, Schwertner HA, Blair SN. The association between physical activity, physical fitness, and Type 2 diabetes mellitus. Compr Ther 2000;26:176-82

6. Ozougwu JC, Olimba $\mathrm{KC}$, Belonwu $\mathrm{CD}$, Unakalamba $\mathrm{CB}$. The pathogenesis and pathophysiology of Type 1 and Type 2 diabetes mellitus. Acad J 2013;4:46-57.

7. Koivisto VA, Defronzo RA. Exercise in the treatment of Type 2 diabetes. Acta Endocrinol 1984;2:107-11.

8. Dela F, Mikines KJ, Sonne B, Galbo H. Effect of training on interaction between insulin and exercise in human muscle. J Appl Physiol (1985) 1994;76:2386-93.

9. Benkar AP, Kanase SB. Effect of aerobic exercises and resistance training on glucose levels in Type 2 diabetic subjects. Asian J Pharm Clin Res 2017;10:360-3.

10. Sree Lekshmi RS, Shanmugasundaram P. A comprehensive review on depression in diabetic patients. Asian J Pharm Clin Res 2017;10:23-30.

11. Kalsi A, Singh S, Taneja N, Kukal S, Mani S. Current treatments for Type 2 diabetes, their side effects and possible complementary treatments. Int J Pharm Pharm Sci 2015;7:13-8.

12. Kartono A, Irawati FD, Setiawan AA, Syafutra H, Sumaryada T. The effects of physical exercise on the insulin-dependent diabetes mellitus subjects using the modified minimal model. Int J Pharm Pharm Sci 2017;9:179-86. 